\title{
Prevalence of lymphoid follicles and aggregates in Helicobacter pylori gastritis in antral and body
}

\section{mucosa}

\author{
S Eidt, $M$ Stolte
}

\begin{abstract}
Aims-To evaluate the prevalence of lymphoid follicles and aggregates in the antral and body mucosa in Helicobacter pylori gastritis and to assess if there were correlations with ulcers in the duodenum, pylorus, or stomach, and with chronic antral erosions.
\end{abstract}

Methods-Patients (n = 2692) with histologically confirmed $H$ pylori antral gastritis were investigated. These comprised five groups: those with duodenal ulcers; those with pyloric ulcers; those with gastric ulcers; those with chronic erosions; and those with no associated lesions. In 1446 cases at least two additional biopsy specimens from the oxyntic mucosa were available.

Results-Lymphoid follicles and aggregates were found in $53.8 \%$ of cases in the antral mucosa compared with $14.8 \%$ in the oxyntic mucosa $(p<0.001)$. The various diseases showed significant differences in terms of the prevalence of follicles and aggregates: The highest numbers in the antral mucosa as well as the lowest in the oxyntic mucosa were found in patients with duodenal ulcers (60.5\% and $9.2 \%$, respectively). The highest numbers of follicles and aggregates in the oxyntic mucosa occurred in patients with gastric ulcers.

Conclusions-The detection of lymphoid follicles and aggregates in oxyntic mucosa and the higher prevalence in antral mucosa fits well with the distribution of primary gastric lymphomas. This adds further weight to the notion that the development of follicles and aggregates, triggered by $H$ pylori, might be an early precursor to gastric lymphoma. The differences between the groups investigated might be due to different strains of $H$ pylori or differences in the respective sizes of antral and oxyntic mucosa.

(F Clin Pathol 1993;46:832-835)

Institute of Pathology, University of Cologne S Eidt

Institute of Pathology, Klinikum Bayreuth, Preuschwitzer Str. 101, D-95445

Bayreuth, Germany

M Stolte

Correspondence to:

Dr M Stolte

Accepted for publication 7 April 1993

The normal gastric mucosa contains very few $T$ cells and almost no $B$ cells in the lamina propria. ${ }^{1}$ In the most common type of gastritis-that induced by Helicobacter pyloricolonisation of the gastric mucosa by $H$ pylori is followed by both a local and systemic immune response..$^{2-5}$ Lymphoid follicles and lymphoid aggregates are repeatedly detected at the base of the antral mucosa in cases of $H$ pylori gastritis. They have not been found, however, in chemically induced gastritis or in normal antral mucosa. ${ }^{2367}$ No data are available regarding the prevalence of lymphoid follicles or lymphoid aggregates in the oxyntic mucosa.

\section{Methods}

A total of 2692 patients were investigated from whom two or three biopsy specimens from the antrum were available. Additional biopsy specimens from the oxyntic mucosa were available in 1446 of these patients. Further prerequisites for inclusion in the study were histologically confirmed $H$ pylori gastritis of the antral mucosa, no treatment with antibiotics and no medication with nonsteroidal anti-inflammatory drugs (NSAIDs).

Tissue sections were stained with haematoxylin and eosin and Warthin-Starry stains. Lymphoid follicles were diagnosed when an aggregation of lymphoid cells at the base of the mucosa showed a germinal centre, while lymphoid aggregates were defined as accumulations of lymphocytes and plasma cells located at the base of the mucosa without evidence of germinal centres.

The degree and activity of the gastritis, as well as the density of $H$ pylori colonisation were evaluated semiquantitatively in accordance with the Sydney system, ${ }^{8}$ as described elsewhere. ${ }^{9-11}$ The infiltration of the gastric mucosa by lymphocytes and plasma cells (degree of gastritis) and polymorphonuclear neutrophils (activity of gastritis) and the density of the $H$ pylori colonisation were graded as follows: $0=$ none, $1=$ minimal, $2=$ low grade, $3=$ medium grade, $4=$ high grade. A mean gastritis score was obtained by adding together the figures for degree and activity in each case and dividing the result by the number of cases in each group of patients. "Chronic antral erosions" were diagnosed when regenerative changes occurred in the mucosa at the border of an erosive lesion.

The diagnosis of duodenal ulcers was based on endoscopic appearances only, whereas pyloric or gastric ulcers were histologically confirmed. In patients with associated lesions the evaluation of gastritis was
Table 1 Age and sex distribution of groups of patients investigated

\begin{tabular}{llll}
\hline & Number & Mean age & Sex ratio \\
\hline Gastritis without lesions & $(\mathrm{n}=1196)$ & $51 \cdot 9$ & $1 \cdot 1: 1$ \\
Chronic erosions & $(\mathrm{n}=227)$ & $54 \cdot 6$ & $1 \cdot 4: 1$ \\
Gastric ulcers & $(\mathrm{n}=154)$ & $56 \cdot 9$ & $1 \cdot 3: 1$ \\
Pyloric ulcers & $(\mathrm{n}=210)$ & $55 \cdot 0$ & $2 \cdot 3: 1$ \\
Duodenal ulcers & $(\mathrm{n}=905)$ & $48 \cdot 6$ & $2 \cdot 1: 1$ \\
\hline
\end{tabular}


Table 2 Prevalence of lymphoid follicles and lymphoid aggregates according to age distribution

$<30 \quad 30-39 \quad 40-49 \quad 50-59 \quad 60-69 \quad 70-79 \quad 80-89 \quad$ Mean

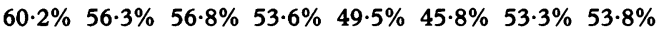

Body $(n=1446)$

made in biopsy specimens obtained distal to the lesions. The patients investigated together with their age and sex distribution are shown in table 1.

Statistical evaluation was done using the $\chi^{2}$.

\section{Results}

Overall, lymphoid follicles and aggregates were present in the antral mucosa of 1447 $(53.8 \%)$ cases and in the oxyntic mucosa of 215 cases $(14.9 \%)$. The differing distribution between antrum and body reached signifi-

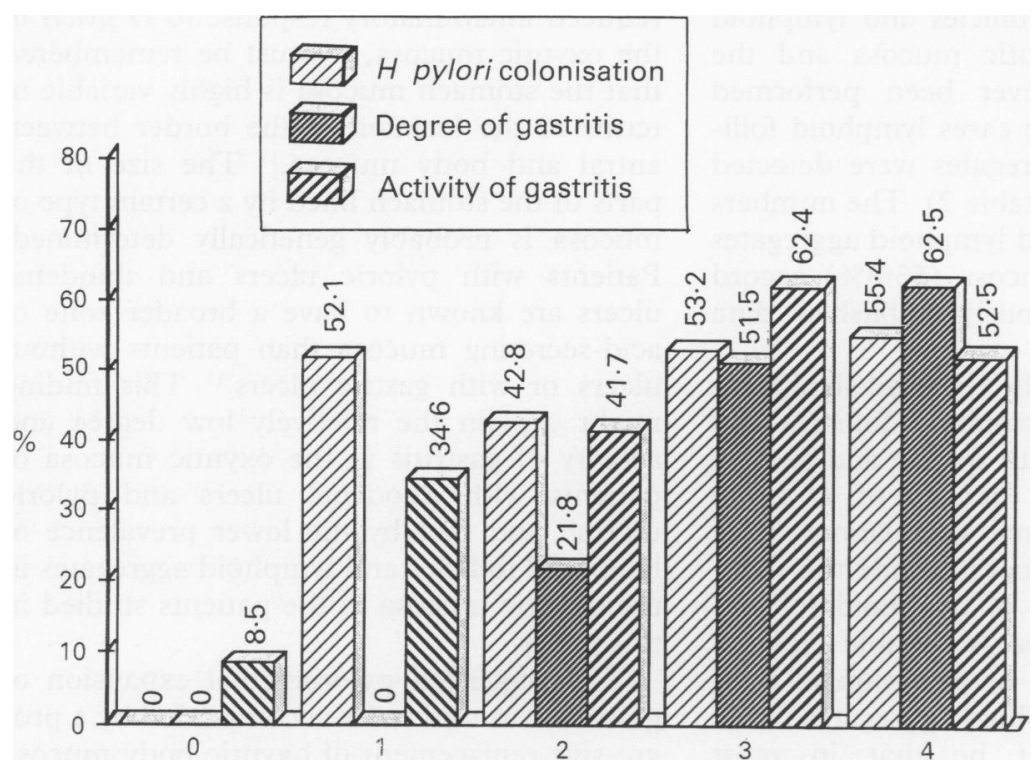

Figure 1 Percentage of cases with detection of lymphoid follicles or lymphoid aggregates correlated with semiquantitatively graded density of $H$ pylori colonisation, activity, and degree of gastritis in the antral mucosa (0 none, 1 minimal, 2 low grade, 3 medium grade, 4 high grade).

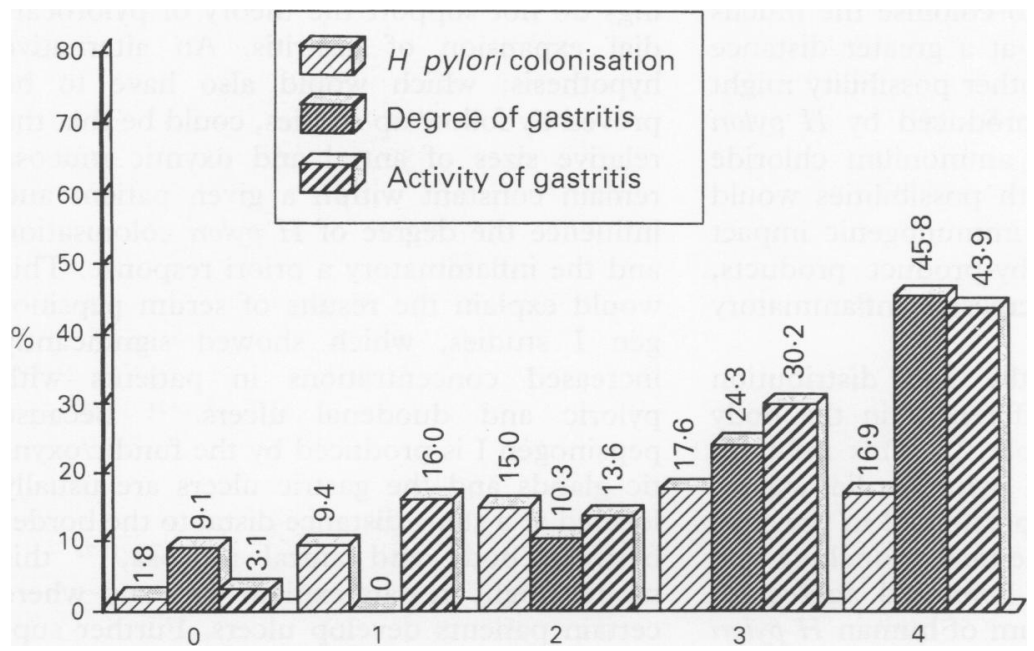

Figure 2 Percentage of cases with lymphoid follicles or lymphoid aggregates correlated with semiquantitatively graded density of $H$ pylori colonisation, activity, and degree of gastritis in the oxyntic mucosa. (O none, 1 minimal, 2 low grade, 3 medium grade, 4 high grade). cance $(\mathrm{p}<0.001)$. Only minor differences were seen in different age groups (table 2).

The correlations between the detection of lymphoid follicles and lymphoid aggregates and the variables of antral gastritis (degree and activity) and the density of $H$ pylori colonisation were evaluated for the overall group (fig 1). With an increasing degree of inflammatory infiltration and increasing density of the $H$ pylori colonisation, higher numbers of lymphoid follicles and lymphoid aggregates were found in the antral mucosa. The same held true in the oxyntic mucosa (fig 2). The correlations reached significance with respect to numbers of lymphoid follicles and lymphoid aggregates and the degree and activity of gastritis in the antral and oxyntic mucosa ( $p<0.001)$, and with density of $H$ pylori colonisation $(\mathrm{p}<0.05$ in the antral mucosa; $\mathrm{p}<0.01$ in the oxyntic mucosa).

The distribution of lymphoid follicles and lymphoid aggregates in the antral mucosa in the five groups of patients is shown in table 3. About two thirds of the patients with duodenal ulcers had evidence of lymphoid follicles and lymphoid aggregates compared with $34.8 \%$ of cases with chronic erosions. An analysis of the prevalence of lymphoid follicles and lymphoid aggregates in the body mucosa showed a similar picture (table 4). Lymphoid follicles and lymphoid aggregates were found in about a quarter of the patients with gastric ulcers compared with $9 \cdot 1 \%$ of those with duodenal ulcers. In all groups, with the exception of those with chronic antral erosions, the prevalence of lymphoid aggregates was higher in the antral than in the oxyntic mucosa. Statistical analysis showed that the different distributions in both types of gastric mucosa were significant with an error probability of less than $0 \cdot 001$.

The mean gastritis score showed small increases in cases with lymphoid follicles and lymphoid aggregates in the antral mucosa compared with those without in all groups, with the exception of patients with chronic

Table 3 Prevalence of lymphoid follicles and lymphoid aggregates in antral mucosa in different groups of patients

\begin{tabular}{lrr}
\hline & \multicolumn{2}{l}{ In the antrum (No/\%): } \\
\cline { 2 - 3 } & lymphoid follicles & lymphoid aggregates \\
\hline Without lesions & $418(34 \cdot 9)$ & $212(17 \cdot 7)$ \\
With chronic erosions & $57(25 \cdot 1)$ & $22(9 \cdot 7)$ \\
With gastric ulcers & $48(31 \cdot 1)$ & $26(16 \cdot 9)$ \\
With pyloric ulcers & $66(32 \cdot 4)$ & $32(15 \cdot 2)$ \\
With duodenal ulcers & $420(46 \cdot 4)$ & $146(16 \cdot 1)$ \\
\hline
\end{tabular}

$\mathrm{p}<0.001$.

Table 4 Prevalence of lymphoid follicles and lymphoid aggregates in oxyntic mucosa in different groups of patients

\begin{tabular}{lll}
\hline & \multicolumn{2}{l}{ In the body (No/\%): } \\
\cline { 2 - 3 } & lymphoid follicles & lymphoid aggregates \\
\hline Without lesions & $55(7 \cdot 8)$ & $67(9 \cdot 5)$ \\
With chronic erosions & $4(6 \cdot 3)$ & $8(12 \cdot 5)$ \\
With gastric ulcers & $17(17 \cdot 5)$ & $6(6 \cdot 2)$ \\
With pyloric ulcers & $5(4 \cdot 7)$ & $10(9 \cdot 3)$ \\
With duodenal ulcers & $20(4 \cdot 3)$ & $23(5 \cdot 0)$ \\
\hline
\end{tabular}

$\mathrm{p}<0.001$ 
Table 5 Comparison of mean gastritis scores in patients with (+) and without (-) lymphoid follicles and aggregates in mucosa of antrum $(A)$ and body $(B)$ of stomach

\begin{tabular}{llllll}
\hline & $A$ & & \multicolumn{2}{l}{$B$} \\
\cline { 2 - 3 } \cline { 5 - 6 } & + & - & & + & - \\
\hline Without lesions & $6 \cdot 1$ & $5 \cdot 3$ & & $5 \cdot 3$ & $3 \cdot 5$ \\
With chronic erosions & $7 \cdot 0$ & $7 \cdot 4$ & & $5 \cdot 3$ & $3 \cdot 9$ \\
With gastric ulcers & $6 \cdot 8$ & $6 \cdot 1$ & & $6 \cdot 3$ & $4 \cdot 7$ \\
With pyloric ulcers & $6 \cdot 6$ & $6 \cdot 0$ & & $5 \cdot 5$ & $4 \cdot 0$ \\
With duodenal ulcers & $6 \cdot 3$ & $6 \cdot 0$ & & $4 \cdot 2$ & $3 \cdot 3$ \\
\hline
\end{tabular}

erosions (table 5). Increases in mean gastritis scores in patients with lymphoid follicles and lymphoid aggregates were present in the oxyntic mucosa in all groups of patients and were greater than those in the antral mucosa.

\section{Discussion}

As far as we are aware, a comparison of the numbers of lymphoid follicles and lymphoid aggregates in the oxyntic mucosa and the antral mucosa has never been performed before. In $14.9 \%$ of the cases lymphoid follicles and lymphoid aggregates were detected in the oxyntic mucosa (table 2). The numbers of lymphoid follicles and lymphoid aggregates found in the antral mucosa $(53.8 \%)$ accord well with other previously published data $(27 \cdot 8 \%-55 \cdot 7 \%) .2367$

Our results confirm the reported significant correlations between lymphoid follicles and lymphoid aggregates with degree and activity of antral gastritis and density of $H$ pylori colonisation. ${ }^{36}$ The same correlations were evident in the body mucosa despite the significantly lower numbers of lymphoid follicles and lymphoid aggregates. A possible explanation for the lower rate of detection of lymphoid follicles and lymphoid aggregates in the oxyntic mucosa might be that in most patients the degree and activity of gastritis is reported to be higher in the antral than in the body mucosa of the same patient. ${ }^{11}$ This might be due to a variety of host or $H$ pylori strain specific factors. The production of hydrochloric acid by the oxyntic mucosa might induce $H$ pylori to colonise the mucus in a lower density and at a greater distance from the mucosa. ${ }^{11}$ Another possibility might be that the ammonia produced by $H$ pylori urease is converted to ammonium chloride hydrochloric acid. ${ }^{12}$ Both possibilities would imply a lesser toxic or immunogenic impact for $H$ pylori and its by-product products, with an attendant decreased inflammatory response. ${ }^{12}$

A comparison of the age distribution showed no relevant differences in the body and antral mucosa (table 2). This militates against the hypothetical pylorocardial expansion of type $B$ gastritis postulated in previous reports. ${ }^{13-15}$ All in all, one might conclude that lymphoid follicles and lymphoid aggregates form part of the spectrum of human $H$ pylori gastritis, both in the antral and body mucosa as has also been observed in animal experiments. ${ }^{16}$

Analysis of the prevalence of lymphoid fol- licles and lymphoid aggregates with respect to the various associated disorders, such as duodenal, pyloric, and gastric ulcers, showed significant differences (tables 3 and 4). In patients with duodenal ulcers the percentage of cases in which lymphoid follicles and lymphoid aggregates were detected was about seven times as high in the antral than the body mucosa. The gastritis scores (table 5) underline the importance of the inflammatory infiltrates. In the antral mucosa the score was $50 \%$ higher than in the body mucosa $(6.3 v$ $4 \cdot 2)$. This finding corroborates earlier results of our group. ${ }^{3}$ Assuming that the development of lymphoid aggregates precedes that of lymphoid follicles, the higher percentage of lymphoid aggregates in the oxyntic mucosa can easily be explained by the less pronounced inflammatory infiltrate compared with that of the antral mucosa. In addition to the hypothetical factors relating to the reduced inflammatory response to $H$ pylori in the oxyntic mucosa, it must be remembered that the stomach mucosa is highly variable in terms of the location of the border between antral and body mucosa. ${ }^{17}$ The size of the parts of the stomach lined by a certain type of mucosa is probably genetically determined. Patients with pyloric ulcers and duodenal ulcers are known to have a broader zone of acid-secreting mucosa than patients without ulcers or with gastric ulcers. ${ }^{15}$ This finding might explain the relatively low degree and activity of gastritis in the oxyntic mucosa of patients with duodenal ulcers and pyloric ulcers $^{18}$ and thereby the lower prevalence of lymphoid follicles and lymphoid aggregates in the oxyntic mucosa in the patients studied in this paper.

The suggested pylorocardial expansion of gastritis was said to be accompanied by a progressive replacement of oxyntic body mucosa by antral mucosa ${ }^{19}$ with an oral shift of the border between the two types of mucosa. ${ }^{13}$ To our knowledge, however, in no paper published to date has a follow up study with topographically standardised biopsy sites been performed. As stated above, our findings do not support the theory of pylorocardial expansion of gastritis. An alternative hypothesis, which would also have to be proved by follow up studies, could be that the relative sizes of antral and oxyntic mucosa remain constant within a given patient and influence the degree of $H$ pylori colonisation and the inflammatory a priori response. This would explain the results of serum pepsinogen I studies, which showed significantly increased concentrations in patients with pyloric and duodenal ulcers. ${ }^{2021}$ Because pepsinogen $\mathrm{I}$ is produced by the fundic/oxyntic glands and the gastric ulcers are usually located at a short distance distal to the border between body and antral mucosa, ${ }^{1322}$ this model might in part explain why and where certain patients develop ulcers. Further support for this hypothesis is gained by the finding that a concomitant $H$ pylori infection had no influence on the different distributions of serum pepsinogen $\mathrm{I}^{20}$ 
An analysis of the group of patients with chronic antral erosions showed the lowest prevalence of lymphoid follicles and lymphoid aggregates in the antral mucosa (table 3 ). We believe that this is due to a relatively recent infection of the gastric mucosa by $H$ pylori. This hypothesis is supported by our unpublished findings of a small percentage of intestinal metaplasia in patients with chronic erosions and a relatively high activity for gastritis in comparison with patients with $H$ pylori gastritis without erosions. ${ }^{23}$ Patients with gastric ulcers had the highest prevalence of lymphoid follicles and lymphoid aggregates in oxyntic mucosa (table 4). Accordingly, the inflammatory score in patients with gastric ulcers was highest in the body mucosa (table 5).

Another interesting point to be considered is the hypothesis of Wotherspoon and coworkers (1991) that infection with $H$ pylori triggers the development of mucosa-associated lymphoid tissue (MALT) in the gastric mucosa, thus providing the necessary substrate on which primary gastric lymphomas, the so-called MALT lymphoma might develop. ${ }^{7}$ As MALT lymphomas occur in all sites in the stomach, with a preference for the antral region, ${ }^{25}$ the similar topographic distribution of lymphoid follicles and lymphoid aggregates found in our study supports that notion. On the other hand, lymphoid follicles and lymphoid aggregates have been found in the surroundings of MALT lymphomas, especially in cases in which infiltration is limited to the mucosa and submucosa. ${ }^{26}$

In conclusion, it might be said that the significant correlations of $H$ pylori colonisation and of histological variables of gastritis in the antral and body mucosa indicate that lymphoid follicles and lymphoid aggregates are an element of the immune response to $H$ pylori in the gastric mucosa as a whole. Because we have been able to show different patterns of gastritis in association with various diseases, it might be speculated whether, by mapping and grading the gastritis, patients at risk for developing gastric or duodenal ulcers may one day be identified. Our findings also support the hypothesis that $H$ pylori gastritis is necessary for the development of MALT and possibly MALT lymphoma. Because MALT lymphomas are rare, even when compared with gastric carcinomas, and $H$ pylori gastritis is common, the risk of a MALT lymphoma developing is small. The endogenous or exogenous factors capable of inducing the development of a lymphomatous proliferation within the setting of $H$ pylori gastritis remain unclear and further investigations to identify them are urgently required.
1 Kirchner T, Melber A, Fischbach W, Heilmann KL, Müller-Hermelink HK. Immunohistological patterns of the local immune response in Helicobacter pylori gastritis. In: Malfertheiner P, Ditschuneit H, eds. Helicobacter pylori, gastritis and peptic ulcers. New York: Springer Verlag, 1990:213-22.

2 Wyatt J, Rathbone BJ. Immune response of the gastric mucosa to Campylobacter pylori. Scand $\mathcal{f}$ Gastroenterol 1988;23(suppl 142):44-9.

3 Stolte M, Eidt S. Lymphoid follicles of the antral mucosa: immune response to Campylobacter pylori? $\mathrm{f}$ Clin Pathol 1989;42:1269-71.

4 Hirschl AM, Rathbone BJ, Berger J, Rotter ML. Four different antigen preparations for serodiagnosis of 67:30-1.

5 Karttunen T, Niemela S. Blood leukocyte count in Campylobacter pylori associated gastritis. Klin Woschenschr 1989;67:33-4.

6 Hauke C, Grabner W, Große M, Stolte M. Zur Frage nach der Lymphfollikelbildung und der Entstehung der intestinalen Metaplasie in der Antrumschleimhaut als Reaktion auf eine Helicobacter pylori- Besiedlung. Leber Magen Darm 1990;4:156-60.

7 Wotherspoon AC, Ortiz-Hidalgo C, Falzon MR, Isaacson PG. Helicobacter pylori-associated gastritis and primary PG. Helicobacter pylori-associated gastritis and prim

8 Price AB. The Sydney system: Histological division. $f$ Gastroenterol Hepatol 1991;6:209-22.

9 Stolte M, Eidt S, Ritter M, Bethke B. Campylobacter pylori und Gastritis. Assoziation oder Induktion? Pathologe 1989;10:21-6.

10 Stolte M, Eidt S, Ohnsmann A. Campylobacter pylori. Unterschiedliche Auswirkungen auf die Magenschleimhaut. In: Ottenjann R, Schmidt $\mathbb{W}$, eds. 2nd, Campylobacter pylori Symposium 1988. Stuttgart: Thieme 1990;38-50.

11 Stolte $M$, Eidt $S$, Ohnsmann A. Differences in Helicobacter pylori associated gastritis in the antrum and body of the stomach. Z Gastroenterol 1990;28:229-33.

12 Triebling AT, Korsten MA, Dlugosz JW, Peronetto F, Lieber CS. Severity of Helicobacter-induced gastric injury correlates with gastric juice ammonia. Dig Dis Sci injury correlates with

13 Stadelmann O, Elster K, Stolte M, et al. The peptic gastric ulcer-histotopographic and functional investigations. Scand $\mathcal{F}$ Gastroenterol 1971;6:613-23.

14 Strickland RG, Mackay IR. A reappraisal of the nature and significance of chronic atrophic gastritis. Dig Dis $S c i$ 1973;18:426-40.

15 Elster $K$. Gastritis aus der Sicht des Pathologen. Arbeitsmed Sozialmed Präventivmed 1978;9:181-3.

16 Krakowka S, Morgan DR, Eaton KA, Radin MJ. Animal models of Helicobacter pylori gastritis. In Menge $\mathbf{H}$, models of Helicobacter pylori gastritis. In Menge H, pregor M, Tytgat GNJ, Marshall BJ, eds: Helicob

17 Elster K, Duschek P, Heinkel K. Ein Beitrag zur Histologie der menschlichen Magenschleimhaut. Zeitschrift für die gesamte innere Medizin 1959;14:728-33.

18 Eidt S, Stolte M. Differences between Helicobacter pylori associated gastritis in patients with duodenal ulcer, pyloric ulcer, other gastric ulcer, and gastritis without ulcer. In: Malfertheiner $\mathrm{P}$, Ditschuneit $\mathrm{H}$, eds. Helicobacter pylori, gastritis and peptic ulcers. New York: Springer Verlag 1990:228-36.

19 Kimura K. Chronological transition of the fundic-pyloric border determined by stepwise biopsy of the lesser and greater curvatures of the stomach. Gastroenterology 1972;63:584-92.

20 Tanaka Y, Mine $K$, Nakai Y, Mishima N, Nakagawa T. Serum pepsinogen I concentrations in peptic ulcer Serum pepsinogen I concentrations in peptic ulcer patients in relation

21 Chang FY, Lai KH, Lee FY, Lee SD, Tsai YT. Serum pepsinogen I levels of gastric ulcer patients are determined by the location of the ulcer crater. Gastroenterol fpn 1992;27:9-14.

22 Oi M, Ito Y, Kumagai F, et al. A possible dual control mechanism in the origin of peptic ulcer. Gastroenterology 1969;57:280-93.

23 Eidt S, Stolte M. Chronische Erosionen der Magenschleimhaut-eine Helicobacter pylori assoziierte Läsion. Verhaudlungen der Deutschen gesellschaft für Pathologie 1990;74:634.

24 Stolte M, Eidt S. Chronic erosions of the antral mucosaa sequelae of Helicobacter pylori-induced gastritis? $Z$ Gastroenterol 1992;30:846-50

25 Eidt S, Stolte $M$. Das maligne Non-Hodgkin Lymphom des Magens. Verdauungskrankheiten 1990;8:90-8.

26 Stolte M, Eidt S. The diagnosis of early gastric lymphoma. Z Gastroenterol 1991;29:6-10. 